\section{Poluição do ar e mortalidade em idosos no Município do Rio de Janeiro: análise de série temporal}

\author{
Air pollution and mortality in the elderly \\ in Rio de Janeiro: a time-series analysis
}

1 Departamento de Epidemiologia, Instituto de Medicina Social, Universidade do Estado do Rio de Janeiro, Rio de Janeiro, Brasil.

Correspondência Regina Paiva Daumas Departamento de Epidemiologia, Instituto de Medicina Social, Universidade do Estado do Rio de Janeiro. Rua São Francisco Xavier 524, 7o andar, Bloco D, Rio de Janeiro, $R J$ 20559-900, Brasil. rpaiva@saude.rj.gov.br

\section{Abstract}

This study investigates the association between particulate air pollution and mortality from cardiovascular and respiratory diseases among the elderly in the city of Rio de Janeiro. Death counts, levels of total suspended particles (TSP), and meteorological data were obtained from secondary databases. Daily counts of deaths and daily TSP levels from 1990 to 1993 were analyzed in Poisson regression models adjusted for long-term temporal trends,seasonality, weekdays, temperature, and humidity. For an increase in TSP levels from the 10th to the 90th percentile $\left(104.7 \mu \mathrm{g} / \mathrm{m}^{3}\right)$, the relative risks for mortality in elderly people from cardiovascular and respiratory diseases were 1.04 (95\%CI: 0.96 1.13) and 1.10 (95\%CI: 0.97-1.26) respectively. Although an upward trend in risk was observed, the associations were not statistically significant, which could be explained partially by the small number of pollution measures available for the period analyzed.

Air Pollution; Cardiovascular Diseases; Respiratory Tract Diseases; Mortality
Regina Paiva Daumas 1

Gulnar Azevedo e Silva Mendonça 1

Antonio Ponce de León 1

\section{Introdução}

A preocupação com os efeitos da poluição atmosférica não é recente, tendo inspirado políticas oficiais de controle já na década de 1950. Naquela época, na Europa, era grande a concentração de indústrias nas grandes cidades e a combustão de carvão era largamente utilizada para aquecimento dos domicílios. Em cidades muito populosas, como Londres, na Inglaterra, os níveis de partículas em suspensão no ar costumavam ser elevados. No episódio que se tornou conhecido como o grande "fog" londrino de 1952, durante uma inversão térmica, o dramático aumento da poluição foi acompanhado de grandes incrementos na mortalidade 1 e na morbidade 2 , principalmente por doenças respiratórias e cardíacas, sobretudo em idosos. Desde então, várias medidas foram tomadas, na Europa e nos Estados Unidos da América, para reduzir a emissão de partículas nas grandes cidades, de tal forma que hoje as concentrações encontradas são cerca de 10 vezes menores que há 50 anos naqueles países 3 .

Apesar dos esforços para reduzir o impacto da poluição urbana na saúde, diversos estudos epidemiológicos continuaram reportando associação significativa entre os níveis de poluição e marcadores de morbidade e mortalidade. Nesses estudos, a poluição por partículas tem sido associada à piora da função pulmonar, ao aumento nos sintomas respiratórios 4 e ao in- 
cremento das internações hospitalares por doenças respiratórias 5,6 e cardiovasculares 7,8. O aumento na mortalidade diária também já foi relatado em muitos países $1,9,10$, podendo estar associado à poluição por partículas, mesmo quando as concentrações médias estão dentro dos padrões internacionais de qualidade do ar 11 .

Nas investigações de efeitos da poluição na saúde, o desenho mais utilizado é o de estudo ecológico de série temporal, trabalhando-se com dados agregados tanto para a exposição, quanto para as informações de saúde. Nesse tipo de estudo são analisadas as co-variações temporais entre a mortalidade e os níveis de poluição urbana, e a inferência sobre a associação depende de se identificar uma relação entre as flutuações na mortalidade diária e os níveis de poluição no mesmo dia ou em alguns dias precedentes. Esta metodologia é destinada, portanto, à mensuração dos efeitos mais imediatos da poluição e não de suas conseqüências a longo prazo 12.

A maioria dos estudos nessa área foi realizada em países do hemisfério norte, onde as condições climáticas e o perfil sócio-demográfico da população são bem distintos dos brasileiros. Além disso, a diversidade de fontes poluidoras, que definem o tamanho e composição das partículas, pode modificar a magnitude da associação entre a poluição e a mortalidade, tornando as funções exposição-resposta variáveis de um país para outro 13,14. Tal fato dificulta a transposição direta para o nosso meio dos achados de estudos norte-americanos e europeus, tornando importante a condução de investigações locais.

Na América Latina, alguns estudos de séries temporais já foram realizados, sendo relatada, dentre outras, associação significativa entre a poluição do ar por partículas e a mortalidade geral nas capitais do México 15 e do Chile 16,17.

No Brasil, na cidade de São Paulo, associação significativa com mortalidade por doenças respiratórias em crianças 19 , internações por doenças respiratórias em crianças 6,20 e mortalidade entre idosos 19,21 já foram relatadas, sugerindo que a poluição atmosférica naquela cidade deve ser considerada como um relevante problema de saúde pública.

No Município do Rio de Janeiro, a segunda maior metrópole do País, a poluição atmosférica também pode representar riscos à saúde, já tendo sido descrita sua associação com a mortalidade infantil por pneumonia 22 . No entanto, os efeitos da poluição do ar sobre a saúde da população desta cidade não têm sido objeto de pesquisa nos últimos anos, havendo necessidade de ampliação e aprofundamento dos es- tudos, com a utilização de metodologias mais adequadas à quantificação destes efeitos.

O objetivo do presente trabalho é investigar a associação entre a poluição do ar por partículas e a mortalidade por causas específicas em idosos no Município do Rio de Janeiro, utilizando um desenho ecológico de série temporal.

\section{Metodologia}

\section{Fontes de dados}

Os dados relativos aos óbitos de residentes no Município do Rio de Janeiro ocorridos no período de 1990 a 1993 e classificados, segundo a causa básica, nos Capítulos de Doenças do Aparelho Circulatório (DAC) (Cód. 390-459) ou Doenças do Aparelho Respiratório (DAR) (Cód. 460-519) da 9a Revisão da Classificação Internacional de Doenças (CID-9) 23 foram fornecidos pela Secretaria Municipal de Saúde 24 em planilhas contendo todos os campos da Declaração de Óbito (DO). Esses dados integram o Sistema de Informação de Mortalidade (SIM), que tem cobertura estimada em $100 \%$ para o município 25. A qualidade da informação das DOs no município também pode ser considerada boa: apenas 7\% do total de óbitos informados nos anos de 1990-1993 ficaram classificados dentro do capítulo Sintomas, Sinais e Afecções Mal Definidas 26. Somente os óbitos de residentes com idade igual ou superior a 65 anos, ocorridos no próprio município, foram selecionados para inclusão no estudo.

Dados sobre poluição por partículas totais em suspensão (PTS) no período de 1990 a 1993 foram fornecidos pela Fundação Estadual de Engenharia do Meio Ambiente 27, que operava, então, com 12 estações manuais no Município do Rio de Janeiro, realizando medições a cada seis dias. A mensuração nesses monitores era realizada pelo método do amostrador de grandes volumes (Hi-vol), que consiste na sucção do ar ambiente através de um filtro por um período de amostragem de 24 horas.

As estimativas de exposição à poluição do ar foram baseadas nas concentrações de PTS ( $\left.\mu \mathrm{g} / \mathrm{m}^{3}\right)$ médias para cada dia. Para o cálculo de médias diárias para o município, foram selecionadas quatro estações de monitoramento que apresentavam maior número de medidas no período: Bonsucesso, Copacabana, Inhaúma e Méier. Objetivando reduzir o número de dias sem médias de poluição, um procedimento de reposição foi adotado, o qual consistiu em estimar, por meio de regressões lineares, as concentrações de PTS de uma estação, consi- 
derando os níveis registrados por outras duas ou três estações. Naqueles dias em que nenhuma ou apenas uma das quatro estações selecionadas teve medidas válidas, as médias de poluição foram consideradas ausentes.

Informações sobre condições meteorológicas foram fornecidas pela Aeronáutica e incluíram medidas diárias de temperatura (mínima, média e máxima) e umidade relativa do ar (média), mensuradas no Aeródromo de Santa Cruz 28. Foram incluídos no banco de dados, para cada dia, os valores de temperatura e umidade do dia corrente (0), do dia anterior (1), de dois dias antes (2), além de médias de dois e três dias anteriores (M01, M12 e M012), para posterior exploração quanto às defasagens que apresentassem o melhor ajuste.

\section{Análise estatística}

Para investigar a associação entre poluição e mortalidade numa série temporal em que os dados de poluição só estavam disponíveis a cada seis dias, a estratégia utilizada neste estudo foi definir as séries de mortalidade também de seis em seis dias. Supondo uma latência de um dia entre a exposição à poluição e o aumento do número de óbitos, foi definida uma série com as contagens de óbitos dos dias consecutivos àqueles em que era mensurada a poluição. Sendo assim, como o primeiro dia da série de poluição era 4 de janeiro de 1990, a série de mortalidade se iniciou no dia 5 de janeiro de 1990, seguindo com os dias 11, 17, 23 e assim por diante. Essa série de mortalidade de seis em seis dias é que foi a base para iniciar a modelagem estatística, fazendo-se, então, o controle para padrões de longo prazo e condições meteorológicas como se faria para uma série temporal diária.

Para cada grupo de causa (DAC e DAR), os dados foram analisados num modelo de regressão de Poisson, com função de ligação logarítmica, tendo como variável dependente o número diário de óbitos. Para possibilitar o ajuste de associações não lineares complexas, foi utilizada a classe de Modelos Aditivos Generalizados, que permite incluir funções não paramétricas ou semiparamétricas das variáveis explicativas 29. Quando se utilizaram funções não paramétricas, um método de regressão local (loess) foi aplicado. O loess é uma generalização do método de médias móveis em que se obtém um valor predito para cada observação através do ajuste de uma regressão linear local ponderada, na qual os pesos decrescem à medida que aumenta a distância da observação de interesse. A conexão dos valores preditos produz uma curva suave cujo grau de suavização é definido por um parâmetro que determina a fração dos dados a ser utilizada para o cálculo da regressão local ou "janela”.

Ajustes para fatores de confusão foram realizados, controlando-se, inicialmente, a tendência temporal e a sazonalidade e, a seguir, os efeitos de dia da semana, feriados, temperatura e umidade. Para tal, foram utilizados procedimentos já padronizados em trabalhos anteriores 30, que são descritos a seguir.

Para ajustar a tendência temporal e a sazonalidade das séries de mortalidade, foram testadas variáveis indicadoras (dummy) para ano e mês, variáveis indicadoras para ano e estação e funções não paramétricas do tempo, do tipo regressão local (loess), com diferentes parâmetros de suavização. Variáveis indicadoras para cada dia da semana foram incluídas no modelo em conjunto sempre que ao menos uma delas fosse estatisticamente significativa.

Após a definição do melhor modelo para controlar os padrões temporais de longo prazo e efeitos de calendário, os resíduos de desvio do modelo foram plotados contra cada variável meteorológica com diferentes defasagens: mesmo dia, dia anterior, dois dias antes e médias de dois ou três dias (0, 1, 2, M01, M12, M012). Esse passo auxiliou na determinação da forma das funções exposição-resposta e na escolha das defasagens com maior efeito. Os padrões exposição-resposta foram modelados, paramétrica ou não parametricamente, de acordo com a forma da associação sugerida e com o critério de informação de Akaike 29. A cada passo da especificação do modelo, gráficos de diagnóstico, como o periodograma e a função de autocorrelação parcial dos resíduos, foram analisados 31. Quando houve sobredispersão dos dados, um modelo de quase-verossimilhança foi adotado.

O modelo central (core model) foi considerado satisfatório quando os resíduos não apresentaram mais associação com as variáveis meteorológicas e a série de resíduos em função do tempo não evidenciou mais nenhum padrão como tendência, sazonalidade ou autocorrelação. Só então as medidas do poluente foram incluídas no modelo, permitindo o cálculo de um coeficiente de regressão para a associação entre PTS e mortalidade, ajustado pelos principais fatores de confusão. A concentração média diária de PTS entrou no modelo na sua forma original, pressupondo uma associação linear entre o logaritmo da mortalidade e os níveis de PTS. Todos os procedimentos estatísticos foram implementados com o programa S-Plus 2000. 


\section{Resultados}

O número de dias em que houve falha no monitoramento das partículas totais em suspensão (PTS) foi elevado. Com medições a cada seis dias, eram esperadas 60 a 61 aferições anuais, totalizando 243 para o período do estudo. No entanto, as medidas efetivamente realizadas não chegaram a 150 em nenhuma das estações (Tabela 1). Após o procedimento de reposição de dados, foi possível estimar médias de PTS no Município do Rio de Janeiro para 179 dias, aproximadamente $74 \%$ do total.

Os níveis de poluição atmosférica foram heterogêneos entre as estações, sendo aceitáveis em Copacabana e no Méier e elevados em Bonsucesso e Inhaúma. Nestas duas últimas, o padrão para média geométrica anual (MGA) de $80 \mu \mathrm{g} / \mathrm{m}^{3}$ foi excedido em todos os anos e houve várias violações ao padrão diário, estabelecido pela legislação ambiental brasileira em $240 \mu \mathrm{g} / \mathrm{m}^{3}$. A correlação entre as medidas de PTS das quatro estações foi boa (variando de 0,54 a 0,72 ) e muito significativa estatisticamente (valor $\mathrm{p}<0,001$ ). Os níveis médios de PTS estimados para o Município do Rio de Janeiro apresentaram flutuação sazonal, sendo geralmente mais elevados nos meses frios. Também estiveram associados com o dia da semana, sendo estatisticamente significante a diferença entre os níveis registrados às segundasfeiras e aos domingos (dados não mostrados).

No período de 1990 a 1993, foram informados pela Secretaria Municipal de Saúde 85.229 óbitos de residentes no Município do Rio de Janeiro, ocorridos no próprio município e classificados, segundo a causa básica, nos capítulos de doenças do aparelho circulatório (DAC) ou respiratório (DAR) da CID-9. A exclusão daqueles em que não havia informação sobre o dia do óbito ou a data de nascimento representou uma perda inferior a $1 \%$. Foram selecionados para análise os óbitos de pessoas com idade igual ou superior a 65 anos, os quais corresponderam a 64,8 e $66,1 \%$ do total de óbitos por DAC e por DAR, respectivamente.

$\mathrm{Na}$ amostra utilizada no estudo, a série temporal com dados de seis em seis dias, a média diária de óbitos entre idosos, por cada grupo de causa, foi de 29,7 para DAC e de 8,04 para DAR (Tabela 2). Um padrão sazonal, com aumento da mortalidade no inverno, pôde ser identificado para ambos os grupos de causa, sendo mais evidente para DAC (Figura 1).

A análise foi realizada utilizando a regressão de Poisson dentro da classe de Modelos Aditivos Generalizados. A tendência temporal e a sazonalidade da série de mortalidade por DAC foram controladas através de uma função não paramétrica (loess) do tempo com um parâmetro de suavização de $20 \%$ dos dados. A curva ajustada pode ser visualizada na Figura 1. Foram incluídas também variáveis indicadoras (dummies) para os dias da semana. Para controlar para os fatores meteorológicos, foram utilizados: o loess da média dos três últimos dias da temperatura mínima, uma variável indicadora para os dias muito quentes e a média da umidade dos dois últimos dias. A variável para os dias quentes era positiva se a média da temperatura máxima dos últimos três dias era maior que $35 \circ$ C. Os resíduos desse modelo não mais mantiveram um padrão temporal evidente (Figura 2), autocorrelação serial estatisticamente significante ou associação com variáveis meteorológicas (dados não apresentados).

Procedimento idêntico ao descrito para DAC foi adotado com a série de mortalidade por DAR, e neste caso as variáveis de controle foram o loess do tempo com parâmetro de suavização de

Tabela 1

Níveis de partículas totais em suspensão (PTS) $\left(\mu \mathrm{g} / \mathrm{m}^{3}\right)$ em cada estação de monitoramento para o período de 1990 a 1993, Rio de Janeiro, Brasil.

\begin{tabular}{|c|c|c|c|c|c|c|c|c|c|c|}
\hline \multirow[t]{2}{*}{ Estação } & \multirow[t]{2}{*}{$\mathbf{n}^{1}$} & \multirow[t]{2}{*}{$\%$} & \multirow[t]{2}{*}{ Média2 } & \multirow[t]{2}{*}{ DP } & \multirow[t]{2}{*}{ Mínimo } & \multicolumn{3}{|c|}{ Percentis } & \multirow[t]{2}{*}{ Máximo } & \multirow{2}{*}{$\begin{array}{c}\text { VPD3 } \\
(\%)\end{array}$} \\
\hline & & & & & & 25 & 50 & 75 & & \\
\hline Bonsucesso & 149 & 61,3 & 221,5 & 82,2 & 51 & 164,5 & 213 & 260 & 531 & 33,0 \\
\hline Copacabana & 134 & 55,1 & 81,4 & 30,5 & 35 & 60 & 76,5 & 98,25 & 227 & 0,0 \\
\hline Inhaúma & 148 & 60,9 & 138,0 & 64,8 & 48 & 94 & 126,5 & 160,8 & 417 & 9,0 \\
\hline Méier & 146 & 60,1 & 75,0 & 33,8 & 27 & 50,8 & 66,5 & 91,3 & 207 & 0,0 \\
\hline
\end{tabular}

1 Número absoluto de medidas e proporção em relação ao total esperado (243).

2 Média aritmética de todas as medidas do período.

3 Dias em que ocorreram violações ao padrão diário de PTS $\left(240 \mu \mathrm{g} / \mathrm{m}^{3}\right)$. 
Estatísticas descritivas para poluição por partículas, variáveis meteorológicas e mortalidade em idosos, por Capítulos da CID, no Município do Rio de Janeiro, Brasil, 1990-19931.

\begin{tabular}{|c|c|c|c|c|c|c|c|c|c|}
\hline & \multirow[t]{2}{*}{ Média } & \multirow[t]{2}{*}{ DP } & \multicolumn{7}{|c|}{ Percentis } \\
\hline & & & Mínimo & 10 & 25 & 50 & 75 & 90 & Máximo \\
\hline PTS (média) & 129,2 & 43,5 & 42,3 & 81 & 99,8 & 122,2 & 148,2 & 187,8 & 282,5 \\
\hline Temperatura ${ }^{2}$ & 24,0 & 3,2 & 15,4 & 19,6 & 21,6 & 24,0 & 26,5 & 28 & 34,1 \\
\hline Umidade & 80,3 & 8,1 & 52 & 70 & 75 & 81 & 86 & 91 & 99 \\
\hline Mortalidade DAC & 29,7 & 7,02 & 16 & 21 & 24 & 30 & 34 & 39 & 52 \\
\hline Mortalidade DAR & 8,04 & 3,35 & 0 & 4 & 6 & 8 & 10 & 12 & 21 \\
\hline
\end{tabular}

1 Dados relativos à amostra incluída no estudo (243 dias).

2 Média diária.

PTS = partículas totais em suspensão; DAC = doenças do aparelho circulatório

$\mathrm{DAR}=$ doenças do aparelho respiratório; DP = desvio padrão.

Figura 1

Série de contagens diárias de óbitos por doenças do aparelho circulatório (DAC) em idosos no Município do Rio de Janeiro, Brasil, no período de 1990 a 1993, com dados de seis em seis dias.

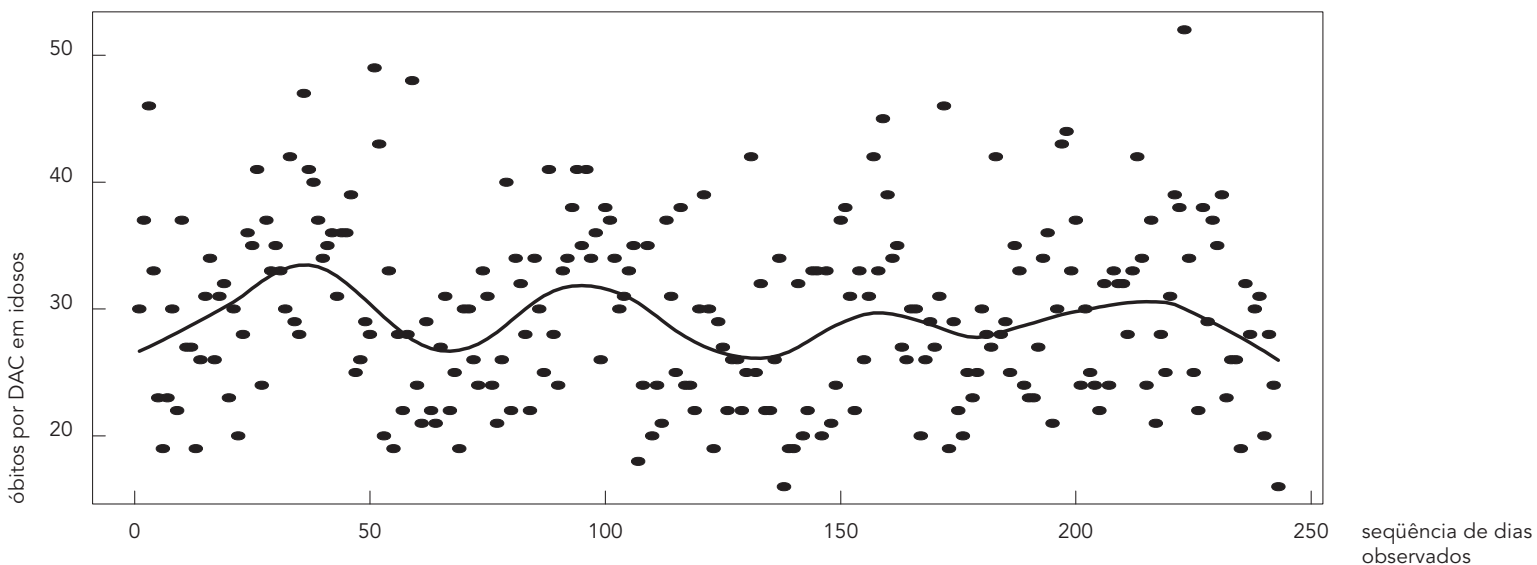

A linha contínua representa uma regressão local ponderada (loess), utilizando uma "janela" que inclu $20 \%$ dos dados: a função não paramétrica que foi incorporada ao modelo para controle da sazonalidade.

18,5\% dos dados, loess da média dos últimos três dias da temperatura máxima e a umidade do dia anterior na forma linear. As variáveis indicadoras para dia da semana não foram estatisticamente significativas no modelo para DAR. A variável indicadora para feriados não foi estatisticamente significante em nenhum dos modelos.

As concentrações de PTS não tiveram associação estatisticamente significante com a mortalidade por DAC ou DAR em idosos. Entretanto, as estimativas pontuais apontaram para um aumento de risco. Uma elevação dos níveis de PTS do 10 o ao 90o percentil $\left(104,7 \mu \mathrm{g} / \mathrm{m}^{3}\right)$ foi associada a um incremento de $4,2 \%$ na mortalidade por DAC e de $10,1 \%$ na mortalidade por DAR, estatisticamente não significativos (Tabela 3).

\section{Discussão}

A mortalidade diária em idosos apresentou flutuação sazonal, com aumento nos meses mais 
Série de resíduos do modelo para mortalidade por doenças do aparelho circulatório (DAC) em idosos ajustado por tendência, sazonalidade, dia da semana, temperatura e umidade.

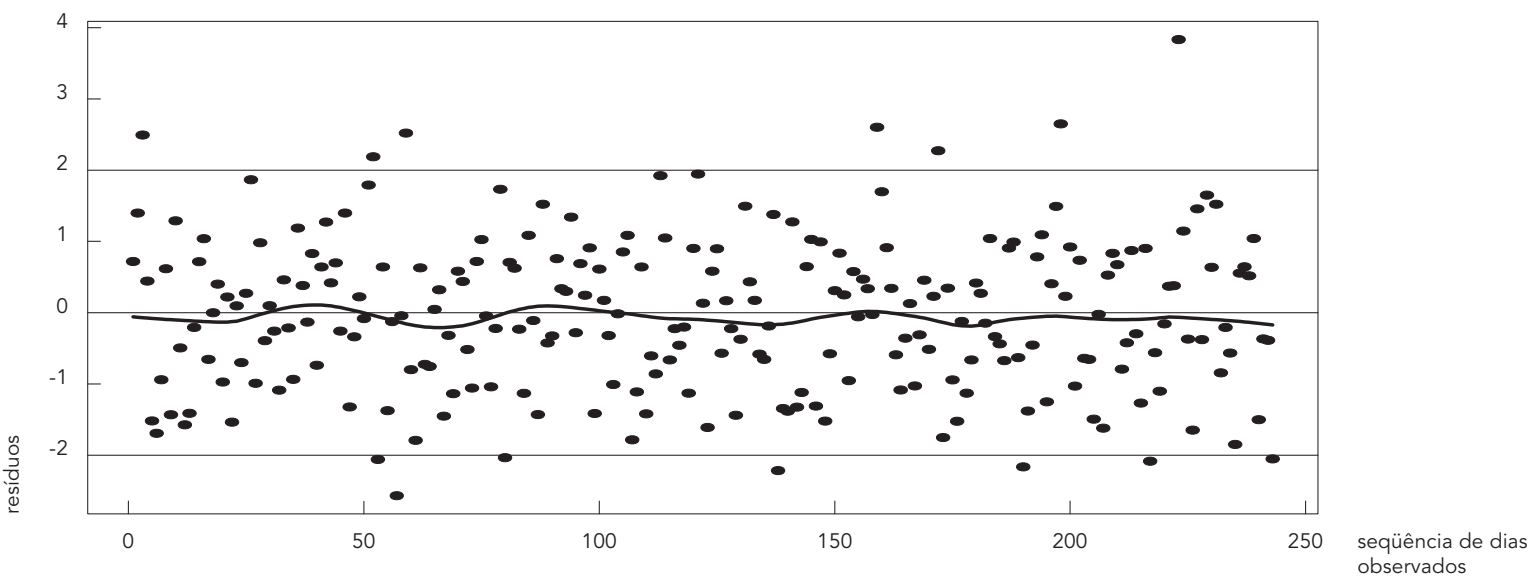

A linha contínua representa uma regressão local ponderada (loess), utilizando uma "janela" que inclui $20 \%$ dos dados.

Tabela 3

Coeficientes de regressão e riscos relativos ajustados da mortalidade em idosos por grupos de causas, para um aumento nos níveis de partículas totais em suspensão do 10 ao 90 percentil.

\begin{tabular}{ccccccc}
\hline & coeficiente & EP & \% RR10-90 & IC95\% & Valor $\mathbf{p}$ \\
\hline DAC & 0,0004 & 0,0004 & 1,042 & 0,960 & 1,130 & 0,33 \\
DAR & 0,0009 & 0,0006 & 1,101 & 0,965 & 1,256 & 0,16 \\
\hline
\end{tabular}

$\mathrm{DAC}=$ doenças do aparelho circulatório; $\mathrm{DAR}=$ doenças do aparelho respiratório $E P=$ erro padrão; $R R=$ risco relativo; $I C=$ intervalo de confiança. frios para ambos os grupos de causa. Parte dessa flutuação pode estar relacionada à maior ocorrência de viroses respiratórias nesses meses 32 , mas um efeito direto das baixas temperaturas também tem sido apontado 33 . No Brasil, na cidade de São Paulo, um aumento significativo da mortalidade por infarto agudo do miocárdio nos meses de inverno já foi descrito 34 , reproduzindo os achados de países de clima temperado. Por outro lado, períodos de calor intenso também se associaram positivamente à mortalidade por DAC, um achado que encontra respaldo em diversos relatos de mortalidade excessiva associada a ondas de calor 35,36.

A associação entre a mortalidade e as condições meteorológicas pode produzir correla- ções espúrias com a poluição do ar. Por esse motivo, os estudos que investigam relações causais entre a poluição do ar e a mortalidade devem ser ajustados de forma adequada por fatores meteorológicos. No presente estudo, para a série de óbitos por DAC, o controle de potenciais fatores de confusão foi bastante satisfatório, com gráficos de diagnóstico apontando para a ausência de correlação com variáveis meteorológicas ou padrões temporais na série de resíduos. Porém, a série de mortalidade por DAR não teve diagnósticos de resíduos tão bons, mesmo após diversas mudanças nas especificações do modelo. Não foi possível, portanto, descartar inteiramente a existência de confundimento residual na análise da mortalidade por DAR.

Quanto à metodologia utilizada para o controle dos fatores de confusão, é importante salientar que, embora o processo de modelagem estatística envolva algum grau de subjetividade, a definição daquele modelo central, com todas as variáveis de controle, antes da inclusão da variável de poluição, garante que tal processo não seja influenciado pelas possíveis associações com a poluição.

Devido à sistemática de realizar as medições de PTS apenas a cada seis dias e em virtude da ocorrência de falhas nas mensurações nas datas previstas, o número de observações na série analisada ficou restrito a 179 dias. Este 
número é bem inferior ao habitual em estudos desse gênero, em que as séries contemplam geralmente três ou mais anos de dados diários. Tais conjuntos de dados podem ser necessários para estimar riscos de pequena magnitude em modelos multivariados. Conseqüentemente, os resultados obtidos não forneceram intervalos de confiança estatisticamente significantes. Todavia, tiveram estimativas pontuais semelhantes às descritas por outros autores para a associação entre a mortalidade geral e PTS 1,15. Especificamente para mortalidade em idosos, estudo realizado em São Paulo por Gouveia \& Fletcher 21 relatou, para uma variação dos níveis de PM10 do 10o ao 90o percentil, um risco relativo de 1,038 (IC95\%: 1,001-1,076) para causas cardiovasculares e de 1,06 (IC 95\%: 1,005$1,118)$ para causas respiratórias. Tais riscos são um pouco menores do que os do presente estudo, porém com intervalos de confiança que incluem os valores aqui encontrados.

É provável que o efeito de alguns dias de poluição atmosférica elevada seja maior do que aquele ocasionado por um único dia de maior exposição. De fato, algumas pesquisas sugerem que os melhores indicadores da exposição seriam as médias de dois a cinco dias precedentes 37 . A ausência de medidas diárias de poluição não permitiu, entretanto, que essa hipótese fosse explorada.

As PTS foram o único poluente do ar analisado no presente estudo, já que não havia dados sobre poluentes gasosos no Rio de Janeiro para aquele período. Por causa da alta correlação entre diversos poluentes atmosféricos, geralmente provenientes de uma fonte comum, o efeito das partículas não pode ser desvinculado dos efeitos de poluentes gasosos. É prudente, portanto, que elas sejam consideradas apenas como uma medida representativa de uma mistura complexa de poluentes das áreas urbanas e não como um agente causal específico dos agravos a elas associados 38 .

O tamanho das partículas é outra questão importante a ser considerada. Quanto menores as partículas, maior a sua capacidade de penetração nas vias aéreas inferiores e alvéolos pulmonares. As chamadas partículas inaláveis, aquelas com diâmetro aerodinâmico menor que 10um (PM10), seriam, assim, a fração das PTS com maior potencial para causar danos à saúde, motivo pelo qual substituíram as PTS na definição do padrão nacional de qualidade do ar da Agência de Proteção Ambiental Americana (EPA), em 1987.

O método de mensuração de PTS pelo amostrador de grandes volumes permite a coleta de partículas de até $50 \mu \mathrm{m}$ de diâmetro. Nessa fai- xa, encontram-se partículas mais grossas, que além de ter menor capacidade de penetração nos pulmões, podem ser de uma origem e composição diferentes das partículas inaláveis. Originam-se, com maior freqüência, por exemplo, da desintegração mecânica do solo, cuja composição é provavelmente menos tóxica que a das partículas produzidas pela indústria e pelos veículos automotores 39. Como a proporção de PM10/PTS é variável de acordo com a localidade e as condições meteorológicas, a mesma concentração de PTS pode representar exposições efetivas diferentes 14. A quantificação da exposição à poluição através da medida de PTS implicaria, por conseguinte, uma perda de precisão.

Desfechos menos graves, como internações, consultas de emergência e sintomas respiratórios, devem ser indicadores mais sensíveis de danos à saúde e precisam ser incorporados às investigações sobre essa questão. Estudos com dados no nível individual, com quantificação seriada da função pulmonar e sintomas respiratórios, por exemplo, podem produzir estimativas mais precisas e ajudar a desvendar os mecanismos fisiopatológicos pelos quais a poluição produz o aumento na mortalidade e na morbidade que se identifica no nível ecológico.

A impossibilidade de mensurar partículas inaláveis e poluentes gasosos, assim como a ausência de medidas diárias de poluição foram limitações importantes deste estudo. Tanto a quantificação da exposição com base nas medidas de PTS, quanto o reduzido número de observações, contribuíram para a perda de precisão nas estimativas de risco, produzindo intervalos de confiança muito alargados. Por esse motivo, os riscos relativos estatisticamente não significativos não devem ser interpretados como indicativos de ausência de associação, e sim como um reflexo de tais limitações.

A instalação recente, no Rio de Janeiro, de estações de monitoramento automáticas, com capacidade para medir diariamente PM10 e diversos poluentes gasosos, permitirá melhor quantificação e caracterização da poluição atmosférica na cidade. Isto tornará possível tanto um controle mais efetivo da emissão de poluentes, quanto a expansão das pesquisas, uma vez que a análise desses dados permitirá estudar os efeitos da poluição com maior precisão e detalhamento.

É importante salientar que, embora os riscos relativos estimados nos estudos sobre poluição sejam geralmente da ordem de 1,01 a 1,10 , a prevenção de riscos de magnitude aparentemente pequena pode ter grande impacto na saúde pública quando se trata de exposi- 
ções muito freqüentes. Por outro lado, os custos envolvidos na redução da emissão de poluentes podem ser bastante elevados quando o objetivo é manter níveis extremamente baixos. Portanto, a quantificação dos efeitos da poluição e a determinação de limites aceitáveis para os níveis de diversos poluentes ambientais são questões fundamentais e que merecem especial atenção nos países em desenvolvimento, os quais convivem não só com níveis de poluição elevados, como também com a escassez de recursos para investimento em medidas de prevenção e controle.

\section{Resumo}

Este artigo tem por objetivo investigar a associação entre a poluição do ar por partículas e a mortalidade por doenças cardiovasculares e respiratórias entre idosos no Município do Rio de Janeiro. Informações relativas à mortalidade, a concentrações de partículas totais em suspensão (PTS) e à meteorologia foram obtidas de fontes de dados secundárias. As contagens diárias de óbitos e os níveis médios diários de PTS para os anos de 1990 a 1993 foram analisados em modelos de regressão de Poisson, que incluíam termos para controle de tendências de longo prazo, flutuações sazonais, dia da semana, temperatura e umidade. Para uma elevação dos níveis de PTS do 10o ao 90opercentil $\left(104,7 \mu \mathrm{g} / \mathrm{m}^{3}\right)$, os riscos relativos para mortalidade por doenças cardiovasculares e respiratórias em idosos foram, respectivamente, 1,04 (IC95\%: 0,96-1,13) e 1,10 (IC95\%:0,97-1,26). Embora tenha sido observada uma tendência de aumento de risco, as associações não foram estatisticamente significativas, o que pode ser atribuído, em parte, ao pequeno número de medidas de poluição disponíveis no período analisado.

Poluição do Ar; Doenças Cardiovasculares; Doenças Respiratórias; Mortalidade

\section{Referências}

1. Schwartz J. Air pollution and daily mortality: a review and meta analysis. Environ Res 1994; 64:3652.

ração deste artigo. G. A. S. Mendonça participou na fundamentação teórica e definição da metodologia. A. P. Leon orientou e supervisionou a análise estatística dos dados. Todos os autores participaram da interpretação e discussão dos resultados.

\section{Agradecimentos}

A M. I. Carvalho, da Fundação Estadual de Engenharia do Meio Ambiente, e C. Noronha, da Secretaria Municipal de Saúde do Rio de Janeiro, pela gentileza na disponibilização dos dados de poluição e mortalidade, respectivamente; a N. Gouveia, da Faculdade de Medicina da Universidade de São Paulo, pelas valiosas sugestões, e a W. L. Junger, pelo auxílio na estruturação dos bancos de dados. Este projeto foi financiado com recursos do Centro Nacional de Epidemiologia, do Ministério da Saúde, no âmbito do projeto VIGISUS e contou com uma bolsa de mestrado da Coordenação de Aperfeiçoamento Pessoal de Nível Superior.
2. Abercrombie GF. December fog in London and the emergency bed service. Lancet 1953; 1:234-5.

3. Schwartz J, Marcus A. Mortality and air pollution in London: a time series analysis. Am J Epidemiol 1990; 131:185-94.

4. Desqueyroux H, Momas I. Pollution atmosphérique et santé: une synthèse des études longitudinales de panel publiées de 1987 a 1998. Rév Épidemiol Santé Publique 1999; 47:361-75.

5. Sunyer J, Spix C, Quenel P, Ponce-de-León A, Ponka A, Barumandzadeh T, et al. Urban air pollution and emergency admissions for asthma in four European cities: the APHEA Project. Thorax 1997; 52:760-5.

6. Gouveia N, Fletcher T. Respiratory diseases in children and outdoor air pollution in Sao Paulo, Brazil: a time series analysis. Occup Environ Med 2000; 57:477-83.

7. Zanobetti A, Schwartz J, Dockery DW. Airborne particles are a risk factor for hospital admissions for heart and lung disease. Environ Health Perspect 2000; 108:1071-7.

8. Poloniecki JD, Atkinson RW, Ponce-de-León A, Anderson HR. Daily time series for cardiovascular hospital admissions and previous day's air pollution in London, UK. Occup Environ Med 1997; 54:535-40. 
9. Anderson HR, Ponce-de-León AC, Bland JM, Bower JS, StrachanDP. Air pollution and daily mortality in London: 1987-92. Br Med J 1996; 312:665-9.

10. Touloumi G, Samoli E, Katsouyanni K. Daily mortality and "winter type" air pollution in Athens, Greece - a time series analysis within the APHEA project. J Epidemiol Community Health 1996; 50 Suppl 1:S47-51.

11. Momas I, Pirard P, Quenel P, Medina S, Le MY, Ferry R, et al. Urban atmospheric pollution and mortality: analysis of epidemiological studies published between 1980 and 1991. Rév Épidemiol Santé Publique 1993; 41:30-43.

12. Schwartz J, Spix C, Touloumi G, Bachárová L, Barumamdzadeh T, Le Tertre A, et al. Methodological issues in studies of air pollution and daily counts of deaths or hospital admissions. J Epidemiol Community Health 1996; 50 Suppl 1:3-11.

13. Aunan K. Exposure-response functions for health effects of air pollutants based on epidemiological findings. Risk Anal 1996; 16:693-709.

14. Romieu I, Borja-Aburto VH. Particulate air pollution and daily mortality: can results be generalized to Latin American countries? Salud Pública Mex 1997; 39:403-11.

15. Borja-Aburto VH, Loomis DP, Bangdiwala SL, Shy CM, Rascon-Pacheco RA. Ozone, suspended particulates, and daily mortality in Mexico City. Am J Epidemiol 1997; 145:258-68.

16. Ostro B, Sanchez JM, Aranda C, Eskeland GS. Air pollution and mortality: results from a study of Santiago, Chile. J Expo Anal Environ Epidemiol 1996; 6:97-114.

17. Cifuentes LA, Vega J, Kopfer K, Lave LB. Effect of the fine fraction of particulate matter versus the coarse mass and other pollutants on daily mortality in Santiago, Chile. J Air Waste Manag Assoc 2000; 50:1287-98.

18. Saldiva PH, Lichtenfels AJ, Paiva PS, Barone IA, Martins MA, Massad E, et al. Association between air pollution and mortality due to respiratory diseases in children in São Paulo, Brazil: a preliminary report. Environ Res 1994; 65:218-25.

19. Saldiva PH, Pope CA, Schwartz J, Dockery DW, Lichtenfels AJ, Salge J, et al. Air pollution and mortality in elderly people: a time-series study in Sao Paulo, Brazil. Arch Environ Health, 1995; 50:159-63.

20. Braga AL, Conceição GM, Pereira LA, Kishi HS, Pereira JC, Andrade MF, et al. Air pollution and pediatric respiratory hospital admissions in São Paulo, Brazil. Journal Environmental Medicine, 1999; 1:95-102.

21. Gouveia N, Fletcher T. Time series analysis of air pollution and mortality: effects by cause, age and socioeconomic status. J Epidemiol Community Health 2000; 54:750-5.

22. Penna MLF, Dulchiade MP. Air pollution and infant mortality from pneumonia in the Rio de Janeiro metropolitan area. Bol Oficina Sanit Panam $1991 ; 25: 47-54$.

23. Organização Mundial da Saúde. Manual da Classificação Internacional de Doenças, Lesões e Causas de Óbito. Nona revisão, 1975. São Paulo: Centro Colaborador da OMS para a Classificação de Doenças em Português; 1978.
24. Secretaria Municipal de Saúde do Rio de Janeiro. Dados sobre mortalidade no Município do Rio de Janeiro. Rio de Janeiro: Coordenadoria de Programas em Epidemiologia, Secretaria Municipal de Saúde; 1999.

25. Paula AM, Evangelista Filho D, Pereira IP, Albano AH, Fernandes RM. Avaliação dos dados de mortalidade, Brasil: 1979 a 1989. Informe Epidemiológico do SUS 1994; 3:21-41.

26. Ministério da Saúde. Mortalidade no Município do Rio de Janeiro. http:/ /www.datasus.gov.br (acessado em 10/Abr/2002).

27. Fundação Estadual de Engenharia do Meio Ambiente. Dados sobre concentrações de partículas totais em suspensão no Estado do Rio de Janeiro: 1990-1997. Rio de Janeiro: Divisão de Qualidade do Ar, Fundação Estadual de Engenharia do Meio Ambiente; 1999.

28. Ministério da Aeronáutica. Dados meteorológicos do Aeródromo de Santa Cruz: 1990-1999. Rio de Janeiro: Divisão de Meteorologia Aeronáutica, Diretoria de Eletrônica e Proteção ao Vôo, Ministério da Aeronáutica; 2000.

29. Hastie T, Tibshirani R. Generalized additive models. London: Chapman \& Hall; 1990.

30. Katsouyanni K, Schwartz J, Spix C, Touloumi G, Zmirou D, Zanobetti A, et al. Short term effects of air pollution on health: a European approach using epidemiologic time series data: the APHEA protocol. J Epidemiol Community Health 1996; 50 Suppl 1:S12-8.

31. Diggle PJ. Time series: a biostatistical introduction. Oxford: Oxford University Press; 1990.

32. Stewart S, McIntyre K, Capewell S, McMurray JJ. Heart failure in a cold climate. Seasonal variation in heart failure-related morbidity and mortality. J Am Coll Cardiol 2002;39:760-6.

33. Aylin P, Morris S, Wakefield J, Grossinho A, Jarup L, Elliott P. Temperature, housing, deprivation and their relationship to excess winter mortality in Great Britain, 1986-1996. Int J Epidemiol 2001; 30:1100-8.

34. Sharovsky R, Cesar LA. Increase in mortality due to myocardial infarction in the Brazilian city of Sao Paulo during winter. Arq Bras Cardiol 2002; 78:106-9.

35. Nakai S, Itoh T, Morimoto T. Deaths from heatstroke in Japan: 1968-1994. Int J Biometeorol 1999; 43:124-7.

36. Dardanoni G, Intonazzo V, La Rosa G, Lanzarone F. Excess deaths related to hot weather in Palermo. Boll Ist Sieroter Milan 1988; 67:251-4.

37. Loomis D, Castillejos M, Gold DR, McDonnell W, Borja-Aburto VH. Air pollution and infant mortality in Mexico City. Epidemiology 1999; 10:118-23.

38. Moolgavkar SH, Luebeck EG. A critical review of the evidence on particulate air pollution and mortality. Epidemiology 1996; 7:420-8.

39. Dockery DW, Pope CA. Acute respiratory effects of particulate air pollution. Annu Rev Public Health 1994; 15:107-32.

Recebido em 16/Dez/2002

Versão final reapresentada em 15/Mai/2003

Aprovado em 20/Nov/2003 